\title{
Erfassung von psychischer Gesundheit und Lebensqualität im Kinder- und Jugendgesundheitssurvey
}

\author{
U. Ravens-Sieberer \\ H. Hölling \\ S. Bettge \\ A. Wietzker
}

\author{
Assessment of Mental Health and Quality of Life in the National Health Interview \\ and Examination Survey for Children and Adolescents
}

\section{Zusammenfassung}

Der Kinder- und Jugendgesundheitssurvey hat das Ziel, für Deutschland verallgemeinerungsfähige, umsetzungsrelevante Daten und Erkenntnisse zur gesundheitlichen Situation von Kindern und Jugendlichen im Altersbereich von 0-17 Jahren zu gewinnen. Dabei kommt der Erfassung der psychischen und subjektiven Gesundheit in Ergänzung der Daten zur körperlichen Gesundheit große Bedeutung zu. Ein nicht geringer Teil der Kinder und Jugendlichen ist von - teilweise lang anhaltenden - psychischen Auffälligkeiten und Erkrankungen betroffen. Subjektive Beeinträchtigungen unter körperlichen, psychischen oder sozialen Aspekten bilden sich in einer verminderten Lebensqualität ab. Nicht zuletzt sind personale, familiäre und soziale Ressourcen der Kinder und Jugendlichen als Schutzfaktoren für psychische und subjektive Gesundheit relevant. Erfahrungen und Ergebnisse aus der Pilotphase des Surveys zeigen, dass die gewählten Erfassungsinstrumente zu psychischen Auffälligkeiten (SDQ), gesundheitsbezogener Lebensqualität (KINDL) und zu Ressourcen (Kombination verschiedener Skalen) für epidemiologische Zwecke geeignet sind und wertvolle Daten liefern können zur Prävalenzschätzung psychischer Auffälligkeiten und Störungen, zur Identifikation von Risikogruppen mit Interventionsbedarf und zur Beschreibung der subjektiven Gesundheit von Kindern und Jugendlichen in Deutschland.

\section{Schlüsselwörter}

Psychische Auffälligkeiten und Störungen · Lebensqualität · Risikound Schutzfaktoren $\cdot$ Kinder und Jugendliche

\begin{abstract}
The National Health Survey for Children and Adolescents plans to collect data on the health status of children and adolescents in the $0-17$ years age bracket which can be used for the generalisation and implementation of measures. In this context, assessment of mental and subjective health, in addition to collection of data concerning somatic health, is of great importance in a survey on the health status of children and adolescents in Germany. The proportion of children and adolescents afflicted by in part long lasting - mental health problems and disorders is considerable. Subjective impairment due to somatic, psychological or social factors result in a reduced quality of life. Last but not least, personal, familial, and social assets of children and adolescents are relevant as protective factors for their mental and subjective health. Experiences and results from the pilot test of the survey demonstrate that the instruments selected for assessing mental health problems (SDQ), health related quality of life (KINDL), and assets (a combination of various scales) are appropriate for epidemiological purposes. They may supply valuable data to estimate the prevalence of mental health problems and psychiatric disorders, to identify risk groups needing intervention, and to describe the subjective health of children and adolescents in Germany.
\end{abstract}

\section{Key words}

Mental health problems and psychiatric disorders · quality of life . risk and protective factors · children and adolescents 


\section{Kenntnisstand und Informationsbedarf}

Für das Kindes- und Jugendalter liegt bisher ein Mangel an aussagefähigen epidemiologischen Daten zum körperlichen Gesundheitszustand vor, ganz besonders aber zur psychischen Gesundheit und zum subjektiven Wohlbefinden [Bundeszentrale für gesundheitliche Aufklärung 1998, Ravens-Sieberer 2000]. Daher erscheint es notwendig, die routinemäßig erfassten Daten durch bevölkerungsbezogene Surveys zu ergänzen, die Daten zur psychischen und subjektiven Gesundheit liefern und deren Verknüpfung mit somatischen Daten erlauben.

Auffälligkeiten und Störungen des Erlebens und Verhaltens im Kindes- und Jugendalter sind häufig und verursachen erhebliche Beeinträchtigungen für die Betroffenen in Familie, Schule und sozialem Umfeld. Die Persistenzrate psychischer Störungen in einem Zeitraum von zwei bis fünf Jahren wird mit über $50 \%$ angegeben [Ihle, Esser 2002]. Bei einem beträchtlichen Anteil psychisch erkrankter Kinder und Jugendlicher ist demnach davon auszugehen, dass es sich hierbei nicht um eine vorübergehende, leicht $\mathrm{zu}$ therapierende Erscheinung handelt, sondern um eine chronische Störung. Entsprechend muss ein gesundheitspolitisches Ziel die Verbesserung der Erkennung und adäquaten Behandlung psychischer Auffälligkeiten von Kindern und Jugendlichen sein, zumal die Wirksamkeit spezifischer Verfahren der Kinder- und Jugendlichenpsychotherapie nachgewiesen werden konnte [Döpfner, Lehmkuhl 2002].

Verlässliche Aussagen über die Prävalenz psychischer Auffälligkeiten und Störungen im Kindes- und Jugendalter sind mittels epidemiologischer Studien schwer zu treffen, da es sich hierbei um eine Vielzahl von Erscheinungsformen mit klinisch aufwändiger Diagnosestellung und einer hohen Komorbiditätsrate handelt. Die Verwendung unterschiedlicher Methodik und Klassifikationssysteme und die Tatsache, dass nur ein geringer Teil der betroffenen Familien professionelle Hilfe in Anspruch nimmt, erschweren diese Aufgabe zusätzlich und lassen Aussagen über die Zunahme oder Abnahme psychiatrischer Störungen über einen Verlauf hinweg gegenwärtig nicht möglich erscheinen [Lehmkuhl et al. 1998]. Schätzungen zum Auftreten psychischer Störungen differieren daher erheblich und weisen im Altersbereich bis 18 Jahre eine Prävalenzrate zwischen 10 und 20\% auf [Petermann et al. 2000]. Der Kinder- und Jugendgesundheitssurvey will hierzu einen Beitrag leisten, indem verlässliche Daten zur Prävalenz psychischer Auffälligkeiten und Störungen im Kindes- und Jugendalter erhoben werden.

Die Bedeutung der subjektiv wahrgenommenen Gesundheit (auch als gesundheitsbezogene Lebensqualität bezeichnet) als ein wesentliches Beschreibungskriterium des Gesundheitszustandes ist vielfach betont worden [z.B. Spilker 1996]. Im Gegensatz zu den klassischen medizinischen Kriterien zur Beurteilung des Gesundheitszustandes einer Person beinhaltet dieses Konzept die für viele Aspekte maßgebliche Sichtweise der Betroffenen hinsichtlich ihrer körperlichen Funktionsfähigkeit und ihres psychischen Wohlergehens [Bullinger, Ravens-Sieberer 1995]. Bisherige Untersuchungen zum Gesundheitszustand und -verhalten von Kindern und Jugendlichen im deutschen Sprachraum haben subjektive Gesundheitsparameter in der Identifikation von Risikopopulationen kaum berücksichtigt; Normdaten zu diesem Themenbereich liegen bisher nicht vor. Die Befragung zur gesundheitsbezogenen Lebensqualität von Kindern und Jugendlichen wurde daher in den Kinder- und Jugendgesundheitssurvey integriert.

In der Entwicklungspsychologie und den Gesundheitswissenschaften werden intensiv die Auswirkungen von Belastungen und Risikofaktoren einerseits und von Ressourcen oder Schutzfaktoren andererseits auf die Gesundheit und das Wohlbefinden diskutiert [Antonovsky 1987]. Unter Schutzfaktoren werden individuelle und soziale Merkmale, Eigenschaften und Lebensumstände verstanden, die sich positiv auf Gesundheit und Wohlbefinden auswirken können. Im Zusammenspiel von biologischen Voraussetzungen und der sozialen und materiellen Umwelt bilden sich beim Kind bzw. Jugendlichen Kompetenzen und Einstellungen heraus, die als personale Ressourcen (oder Schutzfaktoren) für eine erfolgreiche Bewältigung der jeweiligen Entwicklungsaufgaben zu betrachten sind [Masten, Reed 2001].

Mit der Berücksichtigung von Schutzfaktoren für die psychische Gesundheit soll im Survey einer einseitigen Schwerpunktsetzung auf biologische und psychosoziale Risiken (wie z.B. Frühgeburtlichkeit, niedriger sozioökonomischer Status, Migrationshintergrund) entgegengewirkt werden. Aus diesem Grunde werden im Rahmen des Surveys Daten zu sozialen, personalen und familiären Ressourcen als Determinanten der psychischen und subjektiven Gesundheit erhoben. Diese Daten sollen Erkenntnisse zur Ausprägung der Ressourcen, über die Kinder und Jugendliche verfügen, als Schutzfaktoren für eine gesunde psychische Entwicklung und subjektives Wohlbefinden bringen.

\section{Fragestellungen und Methoden}

Zur Ermittlung der Prävalenz psychischer Auffälligkeiten und Störungen bei Kindern und Jugendlichen in Deutschland wird im Kinder- und Jugendgesundheitssurvey der Strengths and Difficulties Questionnaire (SDQ) [Goodman et al. 1998] eingesetzt. Den gängigen psychiatrischen Diagnosesystemen (DSM-IV, ICD-10) folgend, werden dabei Störungsbilder berücksichtigt, die typischerweise im Kindes- und Jugendalter beginnen [Remschmidt, Schmidt 1994, Döpfner et al. 1997]. Die aus England stammende Originalversion des SDQ wurde bereits psychometrisch getestet und validiert. In Studien, die international durchgeführt wurden, zeigt der SDQ zufrieden stellende Eigenschaften als Screening-Instrument [Goodman, Scott 1999, Goodman et al. 2000]. Er umfasst 25 Fragen, die den Subskalen „emotionale Probleme“, „Verhaltensprobleme“, „Aufmerksamkeitsdefizit-/Hyperaktivitätsprobleme“, „Probleme mit Gleichaltrigen“ und „prosoziales Verhalten“ zugeordnet sind. Neben einem Elternurteil für den Altersbereich 4 bis 16 Jahre gibt es eine Selbstberichtsversion für Jugendliche von 11 bis 16 Jahren. Im Kinder- und Jugendgesundheitssurvey wird die Elternversion für den Altersbereich 3 bis 17 Jahre eingesetzt, das Selbsturteil für den Bereich 11 bis 17 Jahre. Die Erfassung psychischer Auffälligkeiten aus der Perspektive verschiedener Beurteiler (Eltern, Kinder, Jugendliche) ist international üblich und bietet eine potenzielle Informationsquelle für mögliches situationsspezifisches Verhalten in Lebensbereichen der Kinder und Jugend- 
lichen, in das gerade die Eltern keinen ausreichenden Einblick (mehr) haben.

Um die Erfassung der zurzeit besonders im Blickpunkt des öffentlichen Interesses stehenden und in ihrer Prävalenz möglicherweise zunehmenden Aufmerksamkeitsdefizit-/Hyperaktivitätsstörungen (ADHD) zu verbessern, wird zusätzlich zu den über die Befragung erhaltenen Angaben die motorische Unruhe der Kinder und Jugendlichen mithilfe eines in den Untersuchungsablauf integrierten Messgeräts „Radaraktometer“ [Huss et al. 1997] objektiv erfasst. Das Gerät basiert auf DopplerRadar und registriert alle Bewegungen in einem definierbaren Aufnahmekegel von 0 bis 5 Metern. Gemessen werden Bewegungen der gesamten Körperoberfläche. Da das Gerät in räumlicher Distanz zu dem Kind steht und keinerlei akustische oder optische Signale aussendet, kann davon ausgegangen werden, dass das kindliche Verhalten durch die Messung nicht beeinflusst wird. Erste Ergebnisse zum Einsatz des Radaraktometers liegen vor und weisen darauf hin, dass diese Messmethode Aufmerksamkeitstests wie dem Continuous-Performance-Test oder vergleichbaren Verfahren überlegen ist [Salbach 2001]. Während der Untersuchungszeit werden die Kinder außerdem in ihrem Verhalten beobachtet, und anschließend wird eine Einschätzung auf der Grundlage einer vierfach gestuften Likert-Skala für die drei Hauptsymptome des ADHD (Störungen der Aufmerksamkeit, Impulsivität und motorische Unruhe) vorgenommen. Die Auswahl der Rating-Items und deren Formulierung orientiert sich an bereits für standardisierte Testsituationen etablierten Beobachtungsverfahren [Swanson 1992], die sich als reliabel und valide erwiesen haben [Roberts 1990]. Die Antwortskala lehnt sich an die beiden Klassiker der ADHD-Forschung (Conners-Skala und die SNAP-IV) an.

Der Katalog psychischer Auffälligkeiten wird im Rahmen des Kinder- und Jugendgesundheitssurveys u. a. ergänzt durch die Erfassung von Kriterien zur Prävalenzschätzung von Essstörungen mit Hilfe des SCOFF-Questionnaire [Morgan et al. 1999]. Weiterhin dienen Altersangaben der Eltern über die Erlangung wesentlicher Meilensteine kindlicher Entwicklung zwischen 0 und 3 Jahren [Frankenburg et al. 1992] einer groben Orientierung über das Vorliegen möglicher Entwicklungsdefizite in den Entwicklungsbereichen Grobmotorik (Sitzen, Gehen), Sprache (erste Worte) und Alltagsfähigkeiten (aus der Tasse trinken).

Als Untersuchungsinstrumentarium zur Erfassung der Lebensqualität dient der Fragebogen KINDL (revidierte Fassung), der in epidemiologischen Untersuchungen bereits als LebensqualitätsScreening-Instrument psychometrisch geprüft wurde [RavensSieberer et al. 2000]. Während die meisten Lebensqualitätsinstrumente für Kinder in englischer Sprache entwickelt und in einem weiteren, methodologisch aufwändigen Schritt übersetzt wurden, liegt mit dem krankheitsübergreifenden KINDL-Fragebogen zur Erfassung der gesundheitsbezogenen Lebensqualität von Kindern und Jugendlichen ein deutschsprachiges Instrument vor (ursprünglich entwickelt von [Bullinger et al. 1994], revidiert von [Ravens-Sieberer, Bullinger 1998]), das bei klinischen Populationen, aber auch bei gesunden Kindern und Jugendlichen eingesetzt werden kann. Der revidierte KINDL ist ein Fragebogen mit 24 Items, der sechs Dimensionen der Lebensqualität (Körper, Psyche, Selbstwert, Familie, Freunde und funktiona- le Aspekte) erfasst. Der KINDL-Fragebogen kommt der Forderung nach Berücksichtigung der kindlichen Entwicklungsfortschritte mit unterschiedlichen Versionen für verschiedene Altersgruppen nach und liegt sowohl in einer Selbstbeurteilungsversion als auch in einer Fremdbeurteilungsversion (Elternurteil) vor.

Die Art und das Ausmaß der Ressourcen, über die Kinder und Jugendliche als Schutzfaktoren für psychische Gesundheit und Lebensqualität verfügen, werden in drei Facetten erfasst: Im Bereich der familiären Ressourcen werden Eltern und Jugendliche in Anlehnung an die Familienklima-Skalen [Schneewind et al. 1985] zum familiären Zusammenhalt (Enge der Bindungen innerhalb der Familie und elterliche Unterstützung) und Familienklima (gemeinsame Aktivitäten, Regeln) befragt. Soziale Ressourcen werden im Selbsturteil der Jugendlichen über die subjektiv wahrgenommene soziale Unterstützung mit der für Jugendliche angepassten deutschen Version der Social Support Scale (SSS) [Donald, Ware 1984] erfasst. Der Aspekt personaler Ressourcen wird über Fragen zum Kohärenzsinn [Kern et al. 1995], zum Optimismus [Grob et al. 1991] sowie zur Selbstwirksamkeitsüberzeugung der Jugendlichen [Jerusalem, Schwarzer 1986] abgebildet.

Ein weiteres, vielfach in den Medien diskutiertes Thema sind sexuelle Übergriffe auf Kinder. Es wird davon ausgegangen, dass Kinder, die Opfer solcher Tätlichkeiten wurden, häufig langfristig unter psychischen Störungen leiden. Schätzungen gehen davon aus, dass bis zu 25\% der erwachsenen Frauen und 5-10\% der Männer als Kinder sexuelle Übergriffe erfahren haben [Herrmann 1998]. Im Kinder- und Jugendgesundheitssurvey wird daher mit der Frage nach derartigen Vorkommnissen versucht, die Häufigkeit des Auftretens sexueller Übergriffe auf Kinder und Jugendliche zu erfassen.

\section{Erfahrungen aus dem Pretest}

Zur Erfassung psychischer Auffälligkeiten in einer bevölkerungsbezogenen epidemiologischen Studie hat sich die deutsche Version des Strengths and Difficulties Questionnaire (SDQ) im Pretest des Kinder- und Jugendgesundheitssurveys als geeignet erwiesen. Die Eltern der 3- bis 17-Jährigen wurden mit dem Elternurteil des SDQ befragt, Jugendlichen im Alter von 11 bis 17 Jahren wurde die Selbstberichtsversion des SDQ vorgelegt. Seine psychometrischen Eigenschaften erweisen sich für die genannten Altersbereiche als gut: Die interne Konsistenz fällt für eine Anzahl von 25 Items für die jeweiligen Gesamtskalen zufrieden stellend aus (Elternurteil: Cronbachs $\alpha=0.81$; Selbsturteil: Cronbachs $\alpha=0.74)$. Der SDQ wird von Eltern und Kindern gleichermaßen gut akzeptiert und zuverlässig beantwortet. Die überwiegende Mehrheit der Kinder und Jugendlichen zeigt sich, wie zu erwarten, im Sinne des SDQ psychisch unauffällig. Selbst- und Elternurteil korrelieren beim Gesamtproblemwert des SDQ hochsignifikant zu $r=0.41$. Die Übereinstimmung der Beurteilung als unauffällig, grenzwertig oder auffällig fällt mit einem Kontingenzkoeffizienten von $r=0.25$ eher gering aus.

Zur Ermittlung der konvergenten Validität wurde der SDQ-Gesamtproblemwert mit verschiedenen somatischen und psychosomatischen Variablen korreliert, von denen angenommen wird, 
dass sie in Wechselwirkung mit der psychischen Gesundheit stehen. Tendenziell haben Kinder, die innerhalb der letzten vier Wochen vor der Befragung unter Schmerzen, vor allem Kopfschmerzen, gelitten haben, höhere SDQ-Gesamtproblemwerte. Dasselbe gilt für weitere Fragen zu persönlichen oder familiären Problemen: Schlafprobleme, problematisches Essverhalten, sexuelle Gewalterfahrungen der Kinder und Jugendlichen sowie aktuelle Belastungen der Eltern korrelieren ebenfalls positiv mit höheren SDQ-Werten. Bis zu 25\% der Varianz der durch den SDQ gemessenen Auffälligkeit wird durch die Fragen nach der therapeutischen Inanspruchnahme erklärt. Je häufiger Kinder und Jugendliche in Beratung oder therapeutischer Behandlung waren, desto höher ist erwartungsgemäß ihr Gesamtwert im SDQ.

Voraussetzung für die Erfassung der Lebensqualität bzw. der subjektiven Gesundheit innerhalb von repräsentativen Surveys ist das Vorhandensein eines Instrumentariums, das methodischen Forderungen als epidemiologisches Instrument nachkommt. Die im Pretest erfolgte Überprüfung der psychometrischen Eigenschaften des KINDL-Lebensqualitätsinstruments bestätigte dessen gute Akzeptanz (96,5\% aller möglichen Selbstangaben und 94,4\% aller möglichen Elternangaben liegen vor), die einerseits die Berechnung des Gesamtwertes bei 98 bis 99\% der Befragten ermöglicht und andererseits eine wichtige Voraussetzung für weitere Gütekriterien darstellt. Die nur geringfügig von einer Normalverteilung abweichende Verteilung der Gesamtrohwerte gewährleistet eine gute Diskriminierbarkeit über den gesamten Bereich der Merkmalsausprägung. Die über die interne Konsistenz ermittelte prinzipielle Messgenauigkeit des KINDL ist mit Werten von Cronbachs $\alpha=0.75$ bei den jüngeren bzw. Cronbachs $\alpha=0.85$ bei den älteren Kindern und Jugendlichen und Cronbachs $\alpha=0.82$ bei den Eltern als zufrieden stellend zu bezeichnen. Einflussfaktoren auf die kindliche Lebensqualität, die bei Anwendung des Instruments zu berücksichtigen sind, beziehen sich auf das Geschlecht und das Alter der Kinder und Jugendlichen, weniger auf die Schichtzugehörigkeit. Jüngere Kinder beschreiben ihre Lebensqualität im Vergleich zu Jugendlichen als besser, und Jungen stufen ihre Lebensqualität höher ein als Mädchen.

Inwieweit der KINDL tatsächlich gesundheitsbezogene Lebensqualität misst, wurde im Pretest, ausgehend von theoretischen Annahmen über das Konstrukt Lebensqualität und dessen $\mathrm{Zu}$ sammenhänge mit anderen Faktoren ermittelt. Die mit chronischen Erkrankungen wie Asthma oder Neurodermitis einhergehenden Lebensqualitätsbeeinträchtigungen schlagen sich, wie zu erwarten, in verminderten Lebensqualitätswerten auf der Gesamtskala sowohl der Kinderversion als auch der Elternversion nieder. Der außerdem von einem guten Lebensqualitätsfragebogen zu erwartende Zusammenhang mit psychischer Auffälligkeit, gemessen mit dem SDQ, lässt sich ebenfalls erkennen: Psychisch auffällige Kinder weisen über sämtliche Subskalen und den Gesamtwert des KINDL (Eltern- und Kinderversion) signifikant niedrigere Werte auf als unauffällige oder grenzwertig klassifizierte Kinder. Während in der Gruppe der 11- bis 13-Jährigen die Elternversion den zu erwartenden Einfluss chronischer Erkrankungen oder psychischer Auffälligkeiten auf die Lebensqualität präziser erfassen kann, verfügt bei den 14- bis 17-Jährigen die Kinderversion über eine höhere Sensibilität für den Einfluss dieser Lebensqualitätsdeterminanten. Der Gesamtscore und die körperliche Skala der Kinderversion korrelieren erwartungsgemäß hoch negativ mit der Schmerzhäufigkeit in den letzten vier Wochen (gesamt: $r_{s}=-0.48$, Körper: $r_{s}=-0.62$ ); d. h., je mehr Schmerzen auftreten, desto geringer wird die Lebensqualität eingeschätzt. Diese Zusammenhänge fallen in der Elternversion niedriger aus (Gesamt: $r_{s}=-0.28$, Körper: $r_{s}=-0.44$ ). Die entsprechenden Validitäten können mit Werten bis 0.62 zum Teil als hoch bezeichnet werden.

Eine Risikogruppe mit mutmaßlich geringer Lebensqualität stellen Kinder und Jugendliche dar, die an langdauernden und belastenden chronischen Erkrankungen wie z.B. Asthma oder Neurodermitis leiden, mehr als fünfmal Schmerzen in den vergangenen vier Wochen hatten und verstärkt institutionelle Gesundheitsdienstleistungen in Anspruch nehmen (mehr als fünf krankheitsbedingte Arztkontakte im letzten Jahr) oder als psychisch auffällig klassifiziert wurden. Im Pretest ist der KINDL-Fragebogen in der Lage, diese Risikogruppe zu identifizieren. Im Vergleich zu anderen Kindern und Jugendlichen zeigt die Gruppe der Risikopersonen eine signifikant geringere Lebensqualität im Gesamtscore des KINDL und den einzelnen Subskalen.

Insgesamt weisen die Ergebnisse auf eine gute Akzeptanz des KINDL-Fragebogens, ausreichende psychometrische Güte und brauchbare Validität und damit die Eignung des Fragebogens zur Erfassung der gesundheitsbezogenen Lebensqualität in Surveys von Kindern und Jugendlichen hin. Vor dem Hintergrund der bisher noch sehr spärlichen Literatur zum Zusammenhang zwischen gesundheitsbezogener Lebensqualität und Risikogruppen von Kindern und Jugendlichen leistet das eingesetzte Fragebogeninstrument einen methodischen Beitrag. Die hier beschriebenen Ergebnisse zeigen, dass der Fragebogen für eine nach externen Kriterien identifizierte Risikogruppe eine deutlich geringere subjektive Gesundheit ermittelt als für unauffällige Kinder und Jugendliche.

Die ausgewählten Skalen zur Erfassung von Ressourcen (als Einflussfaktoren auf psychische Gesundheit und Lebensqualität) erweisen sich im Pretest als psychometrisch brauchbare Instrumente zur Erfassung des Schutzfaktorenkonzepts. Die Fragen wurden von den Probanden gut akzeptiert (Ausfüllraten von 97,7 bis $99,3 \%$ ), so dass bei 98 bis $99 \%$ der Befragten die Skalen gebildet werden konnten. Die Verteilungseigenschaften der Skalen ermöglichen wegen hinreichender Anpassung an die Normalverteilung und geringer Boden- und Deckeneffekte eine gute Differenzierbarkeit über den gesamten Bereich der jeweiligen Schutzfaktorenausprägung. Die prinzipielle Messgenauigkeit kann mit internen Konsistenzen von Cronbachs $\alpha$ bis 0.87 als gut bezeichnet werden. Die Interskalenkorrelation bestätigt die drei Dimensionen des Schutzfaktorenkonzepts, das aus den Faktoren soziale, personale und familiäre Ressourcen besteht.

Die im Rahmen des Pretests durchgeführten Analysen geben starke Hinweise auf die Bedeutung von Schutzfaktoren als Determinanten für die psychische Gesundheit bzw. psychische Auffälligkeiten. Mittels multivariater Regressionsmodelle, die auch soziodemographische Basisvariablen enthalten, können 36-40\% der Varianz in der psychischen Auffälligkeit und 27\% der Varianz gesundheitsbezogener Lebensqualität erklärt werden. Auch eine isolierte Betrachtung einzelner Schutzfaktoren zeigt, dass diese 
bedeutsam mit psychischer Auffälligkeit assoziiert sind (bis $r=0.35$ ). Alle eingesetzten Schutzfaktoren zeigen die zu erwartenden Zusammenhänge mit psychischer Auffälligkeit und genügen einer validen Messung der Ressourcen für psychische Gesundheit und Lebensqualität.

Die anhand der Pretestdaten mittels Verfahren der Item-Response-Theorie durchgeführte Reduktion der Items auf bei Kindern und Jugendlichen einzusetzende und zu Skalen aggregierbare Kernitems führt zu psychometrisch hochwertigen Indikatoren, die in der zu erwartenden Weise mit psychischer Auffälligkeit korrelieren, d.h., psychische Auffälligkeit geht mit geringen sozialen und personalen Ressourcen einher. Auch die bei den älteren Jugendlichen zusätzlich einzusetzende Skala zur Selbstwirksamkeit weist den zu erwartenden Zusammenhang mit psychischer Auffälligkeit auf: Eine geringe Selbstwirksamkeitserwartung geht mit einem höheren Risiko für psychische Auffälligkeit einher. Werden Validitätskoeffizienten berechnet, so erreichen diese mit Werten bis 0.39 eine akzeptable Höhe. Die Gültigkeit (Validität) dieses Schutzfaktorenmessmodells kann damit angenommen werden.

\section{Erwartete Ergebnisse}

Der Einsatz des SDQ in der Hauptphase des Kinder- und Jugendgesundheitssurveys wird Aussagen über die Prävalenz psychischer Auffälligkeiten und Störungen in der Bevölkerungsgruppe der Kinder und Jugendlichen von 3 bis 17 Jahren in Deutschland ermöglichen. Das über die Altersgruppen hinweg einheitliche Erhebungsverfahren gestattet darüber hinaus anhand der querschnittlichen Daten eine Abschätzung der Zuoder Abnahme psychischer Störungen über den Entwicklungsverlauf vom Kleinkindalter bis zum Übergang ins frühe Erwachsenenalter.

Durch die Befragung mit dem KINDL werden repräsentative Normdaten zur gesundheitsbezogenen Lebensqualität von Kindern und Jugendlichen verschiedener Altersstufen und für beide Geschlechter gewonnen, die für die Beurteilung der gesundheitsbezogenen Lebensqualität zusätzlicher im Survey identifizierter klinischer Subgruppen herangezogen werden können. In der Datenanalyse zur Lebensqualität können aus dem Gesamtkollektiv der befragten Studienpopulation die Kinder und Jugendlichen identifiziert werden, die als Risikopopulation angesehen werden müssen und eine erhöhte Interventionsbedürftigkeit aufweisen. Die Kriterien zur Interventionsbedürftigkeit ergeben sich aus den im Kinder- und Jugendgesundheitssurvey zusätzlich erhobenen Teilbereichen wie medizinische, funktionsbezogene und psychosoziale Daten. Es resultieren Aussagen darüber, wie hoch die Prävalenz der Interventionsbedürftigkeit bei Kindern und Jugendlichen in den definierten Altersgruppen ist und hinsichtlich welcher Charakteristika sich interventionsbedürftige und nichtinterventionsbedürftige Kinder und Jugendliche unterscheiden. Mit Hilfe von regressionsanalytischen Modellen können Determinanten der Lebensqualität der Kinder und Jugendlichen identifiziert und auf ihre Bedeutung in identifizierten Subgruppen geprüft werden.
Die Daten zu sozialen, personalen und familiären Ressourcen, die im Rahmen des Surveys erhoben werden, sollen Erkenntnisse zur Ausprägung der Ressourcen bei Kindern und Jugendlichen als Schutzfaktoren für eine gesunde psychische Entwicklung und als Determinanten der Lebensqualität erbringen und Informationen über die Verbreitung von für die psychische Gesundheit relevanten Einflussgrößen liefern. Es ergeben sich hieraus wichtige Informationen für präventive Maßnahmen, die die Basis für künftige gesundheitspolitische Entscheidungen darstellen können.

\section{Literatur}

${ }^{1}$ Antonovsky A. Unraveling the Mystery of Health. San Francisco: Jossey-Bass Publishers 1987

${ }^{2}$ Bullinger M, von Mackensen S, Kirchberger I. KINDL - Ein Fragebogen zur Erfassung der Lebensqualität von Kindern. Z Gesundheitspsychologie 1994; 2: 64-77

3 Bullinger M, Ravens-Sieberer U. Stand der Forschung zur gesundheitsbezogenen Lebensqualität von Kindern. Präv Rehab 1995; 7: 106-121

${ }^{4}$ Bundeszentrale für gesundheitliche Aufklärung (Hrsg). Gesundheit von Kindern. Epidemiologische Grundlagen. Köln: Forschung und Praxis der Gesundheitsförderung 1998; Band 3

${ }^{5}$ Döpfner M, Plück J, Berner W et al. Psychische Auffälligkeiten von Kindern und Jugendlichen in Deutschland - Ergebnisse einer repräsentativen Studie: Methodik, Alters-, Geschlechts- und Beurteilereffekte. Z Kinder Jugendpsychiatr Psychother 1997; 25: 218-233

${ }^{6}$ Döpfner M, Lehmkuhl G. Die Wirksamkeit von Kinder- und Jugendlichenpsychotherapie. Psychol Rundschau 2002; 53: 184-193

${ }^{7}$ Donald CA, Ware JE. The measurement of social support. Research in Community and Mental Health 1984; 4: 325-370

${ }^{8}$ Frankenburg WK, Dodds J, Archer P et al. The Denver II: A major revision and restandardization of the Denver Development Screening Test. Pediatrics 1992; 90: 477-479

${ }^{9}$ Goodman R, Meltzer H, Bailey V. The Strengths and Difficulties Questionnaire: A pilot study on the validity of the self-report version. Eur Child Adolesc Psychiatry 1998; 7: 125-130

${ }^{10}$ Goodman R, Scott S. Comparing the Strengths and Difficulties Questionnaire and the Child Behavior Checklist: Is small beautiful? J Abnorm Child Psych 1999; 27: 17-24

${ }^{11}$ Goodman R, Ford T, Simmons H et al. Using the Strengths and Difficulties Questionnaire (SDQ) to screen for child psychiatric disorders in a community sample. Br J Psychiatry 2000; 177: 534-539

${ }^{12}$ Grob A, Lüthi R, Kaiser FG et al. Berner Fragebogen zum Wohlbefinden Jugendlicher (BFW). Diagnostica 1991; 37: 66-75

${ }^{13}$ Herrmann B. Medizinische Diagnostik bei sexuellem Kindesmißbrauch. Unveröffentl. Manuskript. Klinikum Kassel: Kinderklinik 1998; 2. ergänzte Aufl

${ }^{14}$ Huss M, Ganter M, Lehmkuhl U. Three dimensional Doppler radar assessment of global motor activity synchronized to EEG. Electroenc Clin Neurophysiol 1997; 103: 130

${ }^{15}$ Ihle W, Esser G. Epidemiologie psychischer Störungen im Kindes- und Jugendalter: Prävalenz, Verlauf, Komorbidität und Geschlechtsunterschiede. Psychol Rundschau 2002; 53: 159-169

${ }^{16}$ Jerusalem M, Schwarzer R. Selbstwirksamkeit. In: Schwarzer R (Hrsg). Skalen zur Befindlichkeit und Persönlichkeit. Institut für Psychologie, Freie Universität Berlin 1986; 15-28

${ }^{17}$ Kern R, Rasky E, Noack RH. Indikatoren für Gesundheitsförderung in der Volksschule. Forschungsbericht 95/1 aus dem Institut für Sozialmedizin. Karl-Franzens-Universität Graz 1995

${ }^{18}$ Lehmkuhl G, Döpfner M, Plück J et al. Häufigkeit psychischer Auffälligkeiten und somatische Beschwerden bei vier- bis zehnjährigen Kindern in Deutschland im Urteil der Eltern - ein Vergleich normorientierter und kriterienorientierter Modelle. Z Kinder Jugendpsychiatr Psychother 1998; 26: 83-96

${ }^{19}$ Masten AS, Reed MG. Resilience in Development. In: Snyder CR, Lopez SJ (Hrsg). The Handbook of Positive Psychology. Oxford: University Press 2001

${ }^{20}$ Morgan JF, Reid F, Lacey H. The SCOFF questionnaire: Assessment of a new screening tool for eating disorders. BMJ 1999; 319: 1467-1468 
${ }^{21}$ Petermann U, Döpfner M, Lehmkuhl G et al. Klassifikation und Epidemiologie psychischer Störungen. In: Petermann F (Hrsg). Lehrbuch der klinischen Kinderpsychologie und -psychotherapie. Berlin: Hogrefe 2000; 30-56

${ }^{22}$ Ravens-Sieberer U, Bullinger M. Assessing health related quality of life in chronically ill children with the German KINDL: First psychometric and content-analytic results. Quality of Life Research 1998; 7: 399-407

${ }^{23}$ Ravens-Sieberer U. Verfahren zur Erfassung der gesundheitsbezogenen Lebensqualität bei Kindern und Jugendlichen - ein Überblick. Bundesgesundheitsbl - Gesundheitsforsch - Gesundheitssch 2000; 3: $198-209$

${ }^{24}$ Ravens-Sieberer U, Görtler E, Bullinger M. Subjektive Gesundheit und Gesundheitsverhalten von Kindern und Jugendlichen - Eine Befragung Hamburger Schüler im Rahmen der Schulärztlichen Untersuchung. Gesundheitswesen 2000; 62: 148-155

${ }^{25}$ Remschmidt H, Schmidt MH. Multiaxiales Klassifikationsschema für psychische Störungen des Kindes- und Jugendalters nach ICD-10 der WHO. Bern: Huber 1994; (3. revidierte Auflage)
${ }^{26}$ Roberts MA. A Beharrioral Observation Method for Differentiating Hyperactive and Aggressive Boys. J Abnorm Child Psych 1990; 18: $131-142$

27 Salbach H. Diagnostische Relevanz des Belohnungsaufschubs bei hyperkinetischen Kindern unter Berücksichtigung der motorischen Aktivität. Unveröffentlichte Dissertationsschrift. Medizinische Fakultät der Charité, Humboldt-Universität Berlin 2001

${ }^{28}$ Schneewind K, Beckmann M, Hecht-Jackl A. Familienklima-Skalen. Institut für Psychologie - Persönlichkeitspsychologie und Psychodiagnostik der LMU München Bericht 8.1 und 8.2,1985

${ }^{29}$ Spilker B. Introduction to the field of quality of life trials. In: Spilker B (Hrsg). Quality of Life and Pharmaeconomics in Clinical Trials. Philadelphia: Lippincott-Raven 1996; 1-10

${ }^{30}$ Swanson J. School-based assessment and interventions for ADD students. Irvine CA: K. C. Publishing 1992 